BMJ Open

Diabetes

Research

\& Care

\title{
Type 2 diabetes and the risk of cardiovascular events in peripheral artery disease versus coronary artery disease
}

\author{
Lukas Sprenger (D) ,1,2,3 Arthur Mader, ${ }^{1,2,3}$ Barbara Larcher, ${ }^{1,2,3}$ \\ Maximilian Mächler, ${ }^{1,2,3}$ Alexander Vonbank, ${ }^{1,2,3}$ Daniela Zanolin-Purin,, ${ }^{1,3}$ \\ Andreas Leiherer (1) , ,1,3 Axel Muendlein, ${ }^{1,3}$ Heinz Drexel (1) , $1,3,4,5$ \\ Christoph H Saely ${ }^{1,2,3}$
}

To cite: Sprenger L, Mader A, Larcher B, et al. Type 2 diabetes and the risk of cardiovascular events in peripheral artery disease versus coronary artery disease. BMJ Open Diab Res Care 2021;9:e002407. doi:10.1136/ bmjdrc-2021-002407

Received 27 May 2021 Accepted 18 September 2021

A Check for updates

(C) Author(s) (or their employer(s)) 2021. Re-use permitted under CC BY-NC. No commercial re-use. See rights and permissions. Published by BMJ.

${ }^{1}$ VIVIT, Vorarlberg Institute of Vascular Investigation and Treatment, Feldkirch, Vorarlberg, Austria

2Department of Medicine I, Academic Teaching Hospital Feldkirch, Feldkirch, Vorarlberg, Austria

${ }^{3}$ Private University in the Principality of Liechtenstein, Triesen, Liechtenstein ${ }^{4}$ Drexel University College of Medicine, Philadelphia, Pennsylvania, USA ${ }^{5}$ Department of Medicine, County Hospital Bregenz, Bregenz, Vorarlberg, Austria

Correspondence to Dr Christoph H Saely; christoph.saely@icloud.com

\section{ABSTRACT}

Introduction The prevalence of type 2 diabetes mellitus (T2DM) is higher in peripheral artery disease (PAD) than in coronary artery disease (CAD) patients, and PAD overall confers higher cardiovascular risk than CAD. How cardiovascular risk compares between PAD and CAD patients when analyses are stratified by the presence of type 2 diabetes is unclear and is addressed in the present study.

Research design and methods We prospectively recorded major cardiovascular events (MACE; ie, cardiovascular death, myocardial infarction or stroke) over $10.0 \pm 4.7$ years in 923 patients with stable CAD, of whom $26.7 \%$ had T2DM and in 292 patients with PAD, of whom $42.1 \%$ had T2DM. Four groups were analyzed: CAD patients without diabetes (CAD/T2DM-; $n=677$ ), CAD patients with T2DM (CAD/T2DM+; $n=246), P A D$ patients without diabetes (PAD/T2DM-; $n=169)$ and PAD patients with T2DM (PAD/T2DM+; $n=123)$.

Results The event rate for MACE increased over our four investigated groups: it was lowest in CAD/T2DM- patients (2.52 events per 100 person-years). It was significantly higher in CAD/T2DM+ patients (3.96 events per 100 person-years; $p<0.001$ ), in PAD/T2DM- patients (3.68 events per 100 person-years; $p=0.022$ ), and in PAD/ T2DM+ patients (7.10 events per 100 person-years; $\mathrm{p}<0.001)$, who in turn were at a higher risk than CAD/ $\mathrm{T} 2 \mathrm{DM}+$ or $\mathrm{PAD} / \mathrm{T} 2 \mathrm{DM}-$ patients $(\mathrm{p}=0.001$ and $\mathrm{p}<0.001$, respectively). Cox regression analysis after multivariate adjustment showed that the presence of T2DM ( $\mathrm{HR}=1.44$ (95\% $\mathrm{Cl} 1.09$ to 1.92); $p=0.012$ ) and the presence of PAD versus CAD ( $\mathrm{HR}=1.48(95 \% \mathrm{Cl} 1.15$ to 1.91$) ; \mathrm{p}=0.002)$ were mutually independent predictors of cardiovascular events.

Conclusions In conclusion, our data show that T2DM as well as the presence of PAD versus CAD are mutually independent predictors of MACE. Patients with both PAD and T2DM are at an exceedingly high risk of cardiovascular events.

\section{INTRODUCTION}

Type 2 diabetes mellitus (T2DM) is a paramount risk factor for atherosclerotic

\section{Significance of this study}

What is already known about this subject?

- The prevalence of type 2 diabetes mellitus (T2DM) is higher in peripheral artery disease (PAD) than in coronary artery disease (CAD) patients, and PAD overall confers higher cardiovascular risk than CAD.

What are the new findings?

- Our data show that PAD patients without diabetes are at a similar risk than CAD patients with T2DM. In patients with PAD and T2DM, the risk is exceedingly high.

- The presence of PAD is associated with a higher cardiovascular risk than the presence of non-significant coronary stenoses or of one significant coronary stenosis. Only with 2 or $\geq 3$ significant stenoses, the risk of CAD patients approaches that conferred by PAD.

- T2DM increases the risk of major cardiovascular event in both, CAD and PAD patients. Both among T2DM patients and among non-diabetic subjects the risk conferred by PAD is higher than that conferred by CAD.

How might these results change the focus of research or clinical practice?

$>$ Our data demonstrate the important prognostic differences between different patient populations subsumed in the current European Society of Cardiology (ESC) category of very high risk. If confirmed by other studies, our findings call for a further differentiation among patients categorized as very high risk up to now.

cardiovascular disease, ${ }^{1}$ and the leading cause of death in patients with T2DM is cardiovascular disease. ${ }^{2}{ }^{3}$ Concordantly, T2DM in patients with established cardiovascular disease is highly prevalent. ${ }^{4}$

Atherosclerosis is a systemic disease. However, risk factors may vary across the involved vessel beds. We have published 
previously that the prevalence of type 2 diabetes is even higher in peripheral artery disease (PAD) than in coronary artery disease $(\mathrm{CAD})$ patients. ${ }^{4}$

The presence of CAD, and even more so the presence of PAD, indicates a very high risk of cardiovascular events. This especially holds true in the presence of T2DM: T2DM patients with manifest $\mathrm{CAD}^{56}$ or $\mathrm{PAD}^{7}$ are at an extraordinarily high risk of cardiovascular events. However, it is not known how the cardiovascular risk of PAD patients who do not have T2DM compares with the risk of CAD patients with T2DM because the individual and combined effects of CAD, PAD and T2DM on the event rate have not been studied yet.

The aim of this investigation was to prospectively compare the cardiovascular risk between PAD and CAD patients when analyses are stratified by the presence of T2DM. Four groups of patients were included: (1) patients with CAD who did not have diabetes, (2) patients with both CAD and T2DM, (3) patients with PAD who did not have diabetes and (4) patients with the combination of PAD and T2DM.

\section{METHODS}

For this study, 923 patients with CAD and 292 patients with PAD were enrolled. The recruitment was conducted at the Academic Teaching Hospital Feldkirch, Austria, a central European tertiary care center.

As CAD patients, we included Caucasian patients who were referred for coronary angiography for the evaluation of established or suspected CAD and in whom angiography revealed lumen narrowing diagnostic of CAD. Patients with myocardial infarction in the 3 months prior to baseline and patients who had type 1 diabetes were excluded from recruitment, and patients with a history of myocardial infarction were excluded from the present analysis.

As PAD subjects, we enrolled symptomatic individuals of Caucasian ethnology with an ankle-brachial index $<0.9$ or with previous revascularization of peripheral arteries who underwent routine duplex sonography and in whom PAD was sonographically verified. Exclusion criteria were manifest type 1 diabetes, PAD Fontaine stage IV and history of myocardial infarction.

At baseline, we registered height and weight as well as waist and hip circumferences. In addition, we obtained information on classical cardiovascular risk factors including history of smoking, hypertension, established diabetes and family history of atherosclerotic disease in a standardized interview. Systolic and diastolic blood pressures were measured after a 1 hour rest by Riva-Rocci method under resting conditions in sitting position at the day of hospital admission. Hypertension was defined according to the 2013 European Society of Cardiology (ESC)/ESH guidelines. ${ }^{8}$ T2DM was diagnosed according to ADA clinical practice recommendations. ${ }^{9}$
During the follow-up period, we recorded cardiovascular events and all-cause death. Date and cause of death were collected annually using a national registry (Statistik Austria, Vienna, Austria), hospital registries and telephone contacts. Standardized interviews were performed in 2-year intervals to assess non-fatal events. As primary endpoint, three-point major cardiovascular events (MACEs; ie, cardiovascular death, myocardial infaction or stroke) was used. Furthermore, cardiovascular death (fatal myocardial infarction, sudden cardiac death, mortality from congestive heart failure due to $\mathrm{CAD}$, and fatal ischemic stroke), non-fatal myocardial infarction, non-fatal ischemic stroke were recorded. All potential endpoints were independently adjudicated by two of the authors (CHS and AV) who were blinded to baseline data.

Venous blood samples were collected after an overnight fast of at least 12 hours, in CAD patients before coronary angiography was done. Laboratory measurements were performed from fresh serum samples, as we have described previously. ${ }^{10}{ }^{11}$ Serum levels of C reactive protein, plasma glucose triglycerides, total cholesterol, low density lipoprotein (LDL) cholesterol and high density lipoprotein (HDL) cholesterol were measured with a Cobas Integra 800 (Roche, Basel, Switzerland). Hemoglobin A1c (HbA1c) was determined on a Menarini-Arkray KDK HA 8160 (Arkray KDK, Kyoto, Japan) by high-performance liquid chromatography. Creatinine was measured by a kinetic Jaffe method (Roche, Switzerland) using a Hitachi 717 or 911 or a Cobas Integra 800 (Roche). The Chronic Kidney Disease Epidemiology Collaboration creatinine equation was applied to estimate the glomerular filtration rate (eGFR). ${ }^{12}$

Our main analyses addressed four subgroups of patients: those with CAD but not diabetes, those with both CAD and T2DM, those with PAD but not diabetes and those with PAD and T2DM. Comparisons were also made for PAD versus CAD patients and for T2DM patients versus those who did not have diabetes. Furthermore, the impact of the extent of CAD on the incidence of MACE was addressed. Between group differences of baseline, data were tested for statistical significance with $\chi^{2}$ and Mann-Whitney $U$ tests for categorical and continuous variables as appropriate. Event-free survival was estimated by an actuarial approach. Pairwise comparisons of event-free survival between patient groups were done with the Wilcoxon-Gehan statistic. HRs for the incidence of first cardiovascular events were derived from Cox regression analysis. Results from Cox regression analysis were obtained both univariately and after multivariate adjustment for covariates that may potentially affect cardiovascular outcomes. As such, we included age, gender, body mass index (BMI), history of smoking, arterial hypertension, systolic blood pressure, LDL cholesterol, HDL cholesterol, HbA1c, eGFR, use of statins, ACE inhibitors/ATII receptor blocking agents (ATII RBA) and aspirin/clopidogrel. Interaction 
Table 1 Baseline characteristics

\begin{tabular}{|c|c|c|c|c|c|c|}
\hline & CAD & & & PAD & & \\
\hline & T2DM- & T2DM+ & & T2DM- & T2DM+ & \\
\hline & $\mathrm{n}=677$ & $\mathrm{n}=\mathbf{2 4 6}$ & $P$ value & $n=169$ & $n=123$ & $P$ value \\
\hline Age (years) & $64.4 \pm 10.4$ & $64.9 \pm 10.2$ & 0.658 & $65.4 \pm 9.3$ & $66.7 \pm 9.9$ & 0.442 \\
\hline Male gender (\%) & 70.2 & 69.9 & 0.943 & 68.0 & $74.8 \%$ & 0.210 \\
\hline Diabetes duration (years) & & $5.0 \pm 7.0$ & & & $7.1 \pm 8.7$ & \\
\hline Hypertension (\%) & 80.1 & 85.4 & 0.067 & 91.7 & 92.7 & 0.762 \\
\hline Smoking (\%) & 56.1 & 61.8 & 0.124 & 82.8 & 82.1 & 0.872 \\
\hline Body mass index $\left(\mathrm{kg} / \mathrm{m}^{2}\right)$ & $27.0 \pm 3.9$ & $28.8 \pm 4.5$ & $<0.001$ & $26.2 \pm 4.3$ & $28.3 \pm 4.9$ & $<0.001$ \\
\hline Waist circumference (cm) & $96 \pm 11$ & $102 \pm 11$ & $<0.001$ & $96 \pm 13$ & $103 \pm 14$ & $<0.001$ \\
\hline HbA1c (\%) & $5.7 \pm 0.3$ & $7.2 \pm 1.3$ & $<0.001$ & $5.6 \pm 0.3$ & $7.2 \pm 1.5$ & $<0.001$ \\
\hline Triglycerides (mg/dL) & $139 \pm 90$ & $175 \pm 103$ & $<0.001$ & $135 \pm 78$ & $162 \pm 100$ & 0.180 \\
\hline Total cholesterol (mg/dL) & $213 \pm 46$ & $204 \pm 47$ & 0.005 & $192 \pm 39$ & $182 \pm 52$ & 0.160 \\
\hline LDL cholesterol (mg/dL) & $137 \pm 40$ & $126 \pm 39$ & $<0.001$ & $119 \pm 36$ & $111 \pm 44$ & 0.240 \\
\hline HDL cholesterol (mg/dL) & $55 \pm 16$ & $49 \pm 14$ & $<0.001$ & $57 \pm 18$ & $48 \pm 15$ & $<0.001$ \\
\hline Apolipoprotein A1 (mg/dL) & $154 \pm 28$ & $147 \pm 27$ & 0.001 & $163 \pm 33$ & $148 \pm 34$ & $<0.001$ \\
\hline Apolipoprotein B (mg/dL) & $99 \pm 29$ & $100 \pm 30$ & 0.476 & $82 \pm 21$ & $84 \pm 29$ & 0.934 \\
\hline Systolic blood pressure $(\mathrm{mm} \mathrm{Hg})$ & $137 \pm 19$ & $139 \pm 20$ & 0.346 & $141 \pm 22$ & $143 \pm 25$ & 0.421 \\
\hline Diastolic blood pressure (mm Hg) & $81 \pm 10$ & $81 \pm 12$ & 0.638 & $80 \pm 11$ & $81 \pm 14$ & 0.898 \\
\hline Statins (\%) & 42.2 & 43.5 & 0.734 & 60.9 & 65.9 & 0.391 \\
\hline ACE inhibitors/ATII RBA (\%) & 36.8 & 47.6 & 0.003 & 45.6 & 61.8 & 0.006 \\
\hline Beta receptor blocking agents (\%) & 50.7 & 46.3 & 0.245 & 34.9 & 43.1 & 0.156 \\
\hline Aspirin or clopidogrel (\%) & 66.1 & 69.1 & 0.387 & 82.8 & 83.7 & 0.839 \\
\hline
\end{tabular}

To convert values for fasting plasma glucose to $\mathrm{mmol} / \mathrm{L}$ multiply by 0.0555 , to convert values for triglycerides to $\mathrm{mmol} / \mathrm{L}$ multiply by 0.0113 and to convert values for total cholesterol, LDL cholesterol or HDL cholesterol to mmol/L multiply by 0.0259 . Data are shown as means \pm SD if not denoted otherwise.

ACE, angiotensin converting enzyme; ATII RBA, angiotensin II receptor blocking agents; CAD, coronary artery disease; HbA1c, hemoglobin A1c; HDL, high density lipoprotein; LDL, low density lipoprotein; PAD, peripheral artery disease; T2DM, type 2 diabetes mellitus.

analyses were performed including interaction terms in adjusted Cox regression models. Subgroup analyses were made regarding age categories ( $\geq 65$ years vs $<65$ years) and gender. We applied the Hochberg method for multiple comparisons regarding judgments

Table 2 Cardiovascular events per 100 person-years of follow-up

\begin{tabular}{llllll}
\hline & \multicolumn{2}{l}{ CAD } & & \multicolumn{2}{l}{ PAD } \\
\cline { 2 - 3 } \cline { 6 - 6 } & T2DM- & T2DM+ & & T2DM- & T2DM+ \\
\cline { 2 - 3 } & $\mathbf{n = 6 7 7}$ & $\mathbf{n = 2 4 6}$ & & $\mathbf{n = 1 6 9}$ & $\mathbf{n = 1 2 3}$ \\
\hline 3-point MACE & 2.52 & 3.96 & & 3.68 & 7.10 \\
Vascular mortality & 1.04 & 2.11 & & 1.53 & 3.33 \\
Stroke & 0.76 & 1.03 & & 1.24 & 2.26 \\
MCl & 0.91 & 1.18 & & 1.95 & 2.50 \\
All-cause mortality & 3.18 & 5.05 & & 4.84 & 7.65 \\
\hline
\end{tabular}

$\mathrm{CAD}$, coronary artery disease; $\mathrm{MCl}$, myocardial infarction; PAD, peripheral artery disease; 3-point-MACE, major cardiovascular endpoint (including cardiovascular death, non-fatal myocardial infarction and non-fatal stroke); T2DM, type 2 diabetes mellitus. concerning statistical significance. Results are given as mean (SD) if not denoted otherwise. All statistical analyses were performed with the software package SPSS V.26.0 for Mac OSX (SPSS).

\section{RESULTS}

\section{Baseline characteristics}

From our 923 patients with CAD, 246 (26.7\%) had T2DM and 677 did not have diabetes. Among the 292 PAD patients, $123(42.1 \%)$ had T2DM and 168 did not have type 2 diabetes. The prevalence of T2DM was significantly higher in patients who had PAD than in those with CAD $(\mathrm{p}<0.001)$.

Table 1 summarizes the baseline characteristics of patients with $\mathrm{CAD}$ and of those with PAD with respect to the presence of T2DM. Both among CAD and among PAD patients, BMI, weight, waist circumference and HbAlc and the use of ACE inhibitors/angiotensin receptor blocking agents were significantly higher in patients with T2DM than in patients without diabetes. Triglycerides were higher and total cholesterol, LDL cholesterol and 


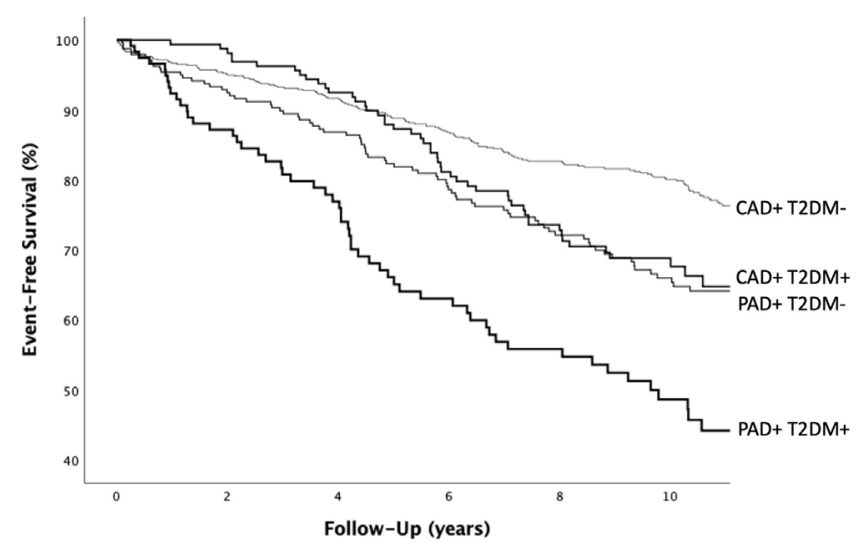

Number at Risk

$\begin{array}{lcccccc} & \text { 0 years } & \mathbf{2} \text { years } & 4 \text { years } & \mathbf{6} \text { years } & \mathbf{8} \text { years } & 10 \text { years } \\ \text { CAD+ T2DM- } & 677 & 618 & 574 & 519 & 452 & 398 \\ \text { CAD+ T2DM+ } & 246 & 217 & 196 & 167 & 138 & 110 \\ \text { PAD+ T2DM- } & 169 & 158 & 145 & 119 & 94 & 59 \\ \text { PAD+ T2DM- } & 123 & 98 & 79 & 61 & 52 & 34\end{array}$

Figure 1 Incidence of MACE. CAD, coronary artery disease; MACE, major cardiovascular event; PAD, peripheral artery disease; T2DM, type 2 diabetes mellitus.

HDL cholesterol were lower in CAD patients who had T2DM than in CAD patients without diabetes.

\section{Cardiovascular events}

During the follow-up period of $10.0 \pm 4.7$ years, 366 MACE (including 163 cardiovascular deaths, 108 nonfatal myocardial infarctions and 95 non-fatal strokes) occurred. The incidence rate of the primary study endpoint thus was $30.0 \%$, corresponding to $3.0 \%$ per year. Table 2 shows the incidence of cardiovascular events and all-cause mortality per 100 person-years of follow-up during the follow-up for the four groups.

In figure 1, the incidence of MACE in the four investigated groups is shown. When compared with the incidence of MACE in CAD+/T2DM- patients (2.52 per 100 person-years), the incidence of MACE was significantly higher in $\mathrm{CAD}+/ \mathrm{T} 2 \mathrm{DM}+$ patients (3.96 events per 100 person-years; $\mathrm{p}<0.001)$, in $\mathrm{PAD}+/ \mathrm{T} 2 \mathrm{DM}-$ patients $(3.68$ events per 100 person-years; $\mathrm{p}=0.022$ ) and in $\mathrm{PAD}+$ /
T2DM+patients (7.10 events per 100 person-years; $\mathrm{p}<0.001)$. Patients with both PAD and T2DM in turn were at a higher risk than $\mathrm{CAD}+/ \mathrm{T} 2 \mathrm{DM}+$ or $\mathrm{PAD}+/ \mathrm{T} 2 \mathrm{DM}-$ patients $(\mathrm{p}=0.001$ and $\mathrm{p}<0.001$, respectively). The incidence of MACE did not differ significantly between $\mathrm{PAD}+/ \mathrm{T} 2 \mathrm{DM}-$ and CAD $+/ \mathrm{T} 2 \mathrm{DM}+$ patients $(\mathrm{p}=0.413)$.

Compared with patients with $\mathrm{CAD}$, Cox regression analysis both univariately and after multivariate adjustment for age, gender, BMI, history of smoking, arterial hypertension, systolic blood pressure, LDL cholesterol, HDL cholesterol, HbA1c, eGFR, use of statins, ACE inhibitors/ATII RBA and aspirin/clopidogrel as well as T2DM showed an increased risk of MACE for the presence of PAD versus that of CAD. Conversely, T2DM increased the risk of MACE after adjustment for age, gender, BMI, history of smoking, arterial hypertension, systolic blood pressure, LDL cholesterol, HDL cholesterol, HbA1c, eGFR, use of statins, ACE-inhibitors/ATII $\mathrm{RBA}$ and aspirin/clopidogrel as well as presence of PAD vs that of CAD. These results are summarized in tables 3 and 4 , respectively.

Furthermore, the presence of PAD versus CAD predicted MACE both among patients with type 2 diabetes and non-diabetic subjects (adjusted HRs 1.22 (95\% CI 0.86 to 1.72 ), $\mathrm{p}=0.274$ and 1.99 (95\% CI 1.35 to $2.92), \mathrm{p}<0.001$; respectively), with a non-significant $\mathrm{p}_{\text {inter- }}$ of 0.235 , indicating that the risk increase conferred by PAD versus $\mathrm{CAD}$ did not differ significantly between T2DM patients and patients without diabetes. Conversely, T2DM predicted MACE both among CAD and among PAD patients (adjusted HRs 1.32 (95\% CI 0.93 to 1.88), $\mathrm{p}=0.121$ and 1.69 (95\% CI 1.00 to 2.86), $\mathrm{p}=0.050$; respectively), with a $\mathrm{p}_{\text {interaction }}$ of 0.121 . Table 5 gives unadjusted as well as adjusted HRs for CAD patients with T2DM, PAD patients without diabetes and PAD patients with T2DM when compared with CAD patients without T2DM.

Patients with $\geq 3 \quad(n=234)$ significant coronary stenoses were at a higher risk of MACE than those who did not have significant stenoses at angiography, with an adjusted HR of 1.58 (95\% CI 1.15 to 2.17 ), $\mathrm{p}=0.005$. The risk of patients with only one significant coronary stenosis $(n=227)$ or two significant stenoses $(n=178)$

Table 3 Results from Cox regression models for T2DM- versus T2DM+

\begin{tabular}{|c|c|c|c|c|}
\hline & HR unadjusted & $P$ value & HR adjusted* & $P$ value \\
\hline 3-point MACE & 1.78 (1.44 to 2.19$)$ & $<0.001$ & 1.44 (1.09 to 1.92$)$ & 0.012 \\
\hline Vascular mortality & 2.17 (1.62 to 2.91$)$ & $<0.001$ & 1.73 (1.15 to 2.60$)$ & 0.008 \\
\hline Stroke & 1.65 (1.13 to 2.40$)$ & 0.009 & 1.20 (0.72 to 2.02$)$ & 0.489 \\
\hline $\mathrm{MCl}$ & 1.46 (1.03 to 2.06$)$ & 0.034 & 1.27 (0.80 to 2.02$)$ & 0.308 \\
\hline All-cause mortality & 1.70 (1.42 to 2.03$)$ & $<0.001$ & 1.29 (1.00 to 1.65$)$ & 0.050 \\
\hline
\end{tabular}

*Adjustments were made for age, gender, BMI, history of smoking, arterial hypertension, systolic blood pressure, LDL cholesterol, HDL cholesterol, HbA1c, eGFR, use of statins, ACE inhibitors/ATII RBA and aspirin/clopidogrel.

ACE, angiotensin converting enzyme; ATII RBA, ATII receptor blocking agents; BMI, body mass index; eGFR, estimated glomerular filtration rate; HbA1c, hemoglobin A1c; HDL, high density lipoprotein; LDL, low density lipoprotein; MCI, myocardial infarction; 3-point MACE, major cardiovascular endpoint (including cardiovascular death, non-fatal myocardial infarction and non-fatal stroke); T2DM, type 2 diabetes mellitus. 
Table 4 Results from Cox regression models for CAD versus PAD

\begin{tabular}{llcll}
\hline & HR unadjusted & P value & HR adjusted* & P value \\
\hline 3-point MACE & $1.78(1.42$ to 2.23$)$ & $<0.001$ & $1.48(1.15$ to 1.91$)$ & 0.002 \\
Vascular mortality & $1.71(1.24$ to 2.36$)$ & 0.001 & $1.33(0.93$ to 1.92$)$ & $1.66(1.07$ to 2.58$)$ \\
Stroke & $2.11(1.42$ to 3.13$)$ & $<0.001$ & $1.98(1.34$ to 2.93$)$ & 0.024 \\
MCl & $2.40(1.68$ to 3.41$)$ & $<0.001$ & $1.32(1.06$ to 1.64$)$ & 0.013 \\
\hline All-cause mortality & $1.80(1.48$ to 2.20$)$ & $<0.001$ & 0.013 \\
\hline
\end{tabular}

*Adjustments were made for age, gender, BMI, history of smoking, arterial hypertension, systolic blood pressure, LDL cholesterol, HDL cholesterol, HbA1c, eGFR, use of statins, ACE-inhibitors/ATII RBA and aspirin/clopidogrel.

ACE, angiotensin converting enzyme; ATII RBA, ATII receptor blocking agents; BMI, body mass index; CAD, coronary artery disease; eGFR, estimated glomerular filtration rate; $\mathrm{HbA1c}$, hemoglobin A1c; HDL, high density lipoprotein; LDL, low density lipoprotein; MCI, myocardial infarction; PAD, peripheral artery disease; 3-point MACE, major cardiovascular endpoint (including cardiovascular death, non-fatal myocardial infarction and non-fatal stroke).

did not differ significantly from those who did not have significant stenoses (HR 1.24 (95\% CI 0.87 to 1.76 ), $\mathrm{p}=0.231$ and 1.34 (95\% CI 0.93 to 1.91 ), $\mathrm{p}=0.113$, respectively). PAD patients were at a significantly higher risk of MACE than patients without significant stenoses and those with a single significant stenosis, with adjusted HRs of 1.92 (95\% CI 1.36 to 2.72), $\mathrm{p}<0.001$ and 1.47 (95\% CI 1.02 to 2.13$), p=0.040$, respectively. The risk of MACE did not differ significantly between PAD patients and CAD patients with $2(\mathrm{p}=0.073)$ or $\geq 3 \quad(\mathrm{p}=0.798)$ significant coronary stenoses.

HRs for the risk increase conferred by the presence of PAD vs that of CAD were 1.94 (95\% CI 1.27 to 2.96), $\mathrm{p}=0.002$ in patients $<65$ years and $1.28(95 \%$ CI 0.93 to $1.75), \mathrm{p}=0.129$ in those $\geq 65$ years of age $\left(\mathrm{p}_{\text {interaction }}=0.270\right)$. Similarly, the impact of T2DM in cardiovascular risk did not differ significantly between patients $<65$ years (HR 2.26 (95\% CI 1.41 to 3.63 ), $p=0.001$ ) and those $\geq 65$ years (HR $1.12(95 \%$ CI 0.75 to 1.66$), \mathrm{p}=0.591), \mathrm{p}_{\text {interaction }}=0.055$.
Regarding gender specific analyses, adjusted HRs for the risk increase conferred by the presence of PAD versus that of CAD were 1.59 (95\% CI 1.19 to 2.12), $p=0.002$ in men and 1.08 (95\% CI 0.63 to 1.85 ], $\mathrm{p}=0.769$ in women $\left(\mathrm{p}_{\text {interaction }}=0.357\right)$. For T2DM, adjusted HRs were 1.52 (95\% CI 1.09 to 2.12$) ; \mathrm{p}=0.014$ in men and 1.36 (95\% CI 0.75 to 2.46$) ; \mathrm{p}=0.313$ in women, with a $\mathrm{p}_{\text {interaction }}$ of 0.135 .

\section{DISCUSSION}

From our results, we conclude that T2DM increases the risk of future MACE in both $\mathrm{CAD}$ and PAD patients. Both among T2DM patients and among non-diabetic subjects the risk conferred by PAD was higher than the risk conferred by CAD.

It is well established that PAD is associated with an even stronger increase in cardiovascular event risk than $\mathrm{CAD},{ }^{13-17}$ but no previous investigation directly adressed single and joint effects of CAD, PAD and T2DM on the

Table 5 Results from Cox regression models in subgroups considering both the presence of CAD versus PAD and the presence of T2DM

\begin{tabular}{|c|c|c|c|c|}
\hline & \multicolumn{2}{|l|}{ CAD } & \multicolumn{2}{|l|}{ PAD } \\
\hline & T2DM- & T2DM+ & T2DM- & T2DM+ \\
\hline 3-point MACE & - & 1.57 (1.21 to 2.03$)$ & 1.50 (1.09 to 2.06$)$ & 2.94 (2.18 to 3.97$)$ \\
\hline Vascular mortality & - & $2.00(1.40$ to 2.85$)$ & $1.46(0.91$ to 2.33$)$ & $3.22(2.13$ to 4.89$)$ \\
\hline Vascular mortality* & - & 1.70 (1.08 to 2.70$)$ & 1.30 (0.78 to 2.16$)$ & 2.13 (1.25 to 3.63$)$ \\
\hline Stroke* & - & 1.05 (0.57 to 1.92$)$ & $1.47(0.82$ to 2.61$)$ & $2.00(1.02$ to 3.91$)$ \\
\hline $\mathrm{MCl}$ & - & $1.32(0.84$ to 2.08$)$ & $2.32(1.48$ to 3.65$)$ & $3.03(1.86$ to 4.95$)$ \\
\hline $\mathrm{MCl}^{*}$ & - & 1.17 (0.68 to 2.01$)$ & $1.88(1.15$ to 3.07$)$ & $2.44(1.33$ to 4.49$]$ \\
\hline All-cause mortality & - & $1.60(1.29$ to 2.00$)$ & $1.69(1.29$ to 2.20$)$ & 2.67 (2.04 to 3.48$)$ \\
\hline
\end{tabular}

*Adjustments were made for age, gender, BMI, history of smoking, arterial hypertension, systolic blood pressure, LDL cholesterol, HDL cholesterol, HbA1c, eGFR, use of statins, ACE-inhibitors/ATII RBA and aspirin/clopidogrel.

ACE, angiotensin converting enzyme; ATII RBA, ATII receptor blocking agents; BMI, body mass index; CAD, coronary artery disease; eGFR, estimated glomerular filtration rate; $\mathrm{HbA1c}$, hemoglobin A1c; HDL, high density lipoprotein; LDL, low density lipoprotein; MCl, myocardial infarction; PAD, peripheral artery disease; 3-point MACE, major cardiovascular endpoint (including cardiovascular death, non-fatal myocardial infarction and non-fatal stroke); T2DM, type 2 diabetes mellitus. 
risk of cardiovascular events. Our data show that PAD patients without diabetes are at a similar risk than CAD patients with diabetes. In patients with PAD and T2DM, the risk is exceedingly high.

The risk of cardiovascular events among CAD patients increases with an increasing extent of CAD ${ }^{18-20}$ We here report the novel finding that the presence of PAD is associated with a higher cardiovascular risk than the presence of non-significant coronary stenoses or of one significant stenosis, only with 2 or $\geq 3$ significant stenoses, the risk of CAD patients approached that of those with PAD. Thus, when considering the risk conferred by CAD, it is important to take into account the severity of the disease. In line with previous publications, ${ }^{21-23}$ we here took into account the presence of significant coronary stenoses $\geq 50 \%$ and the extent of $\mathrm{CAD}$, defined as the number of such significant stenoses. Importantly, we investigated patients with stable CAD. The risk conferred by CAD is expected be higher when patients with acute coronary syndromes are addressed.

These findings have important clinical implications. Screening for PAD using ankle-brachial index measurements is recommended in current ESC guidelines as an inexpensive and broadly available risk stratification tool. This holds true particularly also for a majority of patients with T2DM, among whom PAD is one of the most common manifestations of macrovascular disease. ${ }^{24-27}$ Already non-diabetic CAD patients (and, even, most T2DM patients without CAD) are considered to be at a very high risk of cardiovascular events according to current ESC guidelines and should meet an LDL cholesterol treatment goal of at least $<55 \mathrm{mg} / \mathrm{dL} .{ }^{28}$ Our data demonstrate the important prognostic differences between different patient populations subsumed in the current ESC category of very high risk. If confirmed by other studies, our findings call for a further differentiation among patients categorized as very high risk up to now.

Important strengths of this investigation are the angiographic verification of $\mathrm{CAD}$, the sonographic verification of $\mathrm{PAD}$, and the meticulous acquisition of endpoints. The study applies a hard clinical endpoint that is broadly used in current clinical trials. A potential limitation may be that PAD has not been excluded in all patients with CAD if no signs or symptoms of PAD were observed. Whatsoever, the presence of clinically relevant $\mathrm{PAD}$ in the CAD population would not have created significant differences between the two groups but in contrary would have reduced the observed differences between $\mathrm{CAD}$ and $\mathrm{PAD}$ patients.

In conclusion, our data show that type 2 diabetes and the presence of PAD versus CAD are mutually independent predictors of cardiovascular events. Patients with both PAD and type 2 diabetes are at an exceedingly high risk of cardiovascular events.

Contributors LS: data evaluation and statistical analysis and drafting of the manuscript. AMa: data evaluation and statistical analysis and revision of the manuscript for intellectually important content. BL, MM, AV, DZ-P, AL and AMu: data evaluation and statistical analysis, revision of the manuscript for intellectually important content. HD and CHS: conception and design of the study, data evaluation and statistical analysis, revision of the manuscript for intellectually important content. LS and CHS are responsible for the content of the article.

Funding This work was supported by the Jubiläumsfonds of the Austrian National Bank (project number 14159), Dr. Karl Josef Hier and the Peter Goop Stiftung (Vaduz, Liechtenstein), the Land Vorarlberg and the Institute for Clinical Chemistry at the Academic Teaching Hospital Feldkirch.

Disclaimer These institutions did not have any role in the design of the study and collection, analysis, interpretation of data nor in writing the manuscript.

Competing interests None declared.

Patient consent for publication Not applicable.

Ethics approval The study protocol conforms to the 1975 Declaration of Helsinki and it was approved by the Ethics Committees of the Universities of Innsbruck (Number UN1498 and UN2320) and of the Land Vorarlberg (Number 2006-5/1). All participants gave written informed consent.

Provenance and peer review Not commissioned; externally peer reviewed.

Data availability statement Data are available on reasonable request.

Open access This is an open access article distributed in accordance with the Creative Commons Attribution Non Commercial (CC BY-NC 4.0) license, which permits others to distribute, remix, adapt, build upon this work non-commercially, and license their derivative works on different terms, provided the original work is properly cited, appropriate credit is given, any changes made indicated, and the use is non-commercial. See: http://creativecommons.org/licenses/by-nc/4.0/.

ORCID IDs

Lukas Sprenger http://orcid.org/0000-0003-3798-048X

Andreas Leiherer http://orcid.org/0000-0003-3776-5704

Heinz Drexel http://orcid.org/0000-0002-4677-9756

\section{REFERENCES}

1 Low Wang CC, Hess CN, Hiatt WR, et al. Clinical update: cardiovascular disease in diabetes mellitus: atherosclerotic cardiovascular disease and heart failure in type 2 diabetes mellitus mechanisms, management, and clinical considerations. Circulation 2016;133:2459-502.

2 Andersson T, Hjerpe P, Carlsson AC, et al. Mortality trends and cause of death in patients with new-onset type 2 diabetes and controls: a 24-year follow-up prospective cohort study. Diabetes Res Clin Pract 2018;138:81-9.

3 Einarson TR, Acs A, Ludwig C, et al. Prevalence of cardiovascular disease in type 2 diabetes: a systematic literature review of scientific evidence from across the world in 2007-2017. Cardiovasc Diabetol 2018;17:83.

4 Silbernagel G, Rein P, Saely $\mathrm{CH}$, et al. Prevalence of type 2 diabetes is higher in peripheral artery disease than in coronary artery disease patients. Diab Vasc Dis Res 2015;12:146-9.

5 Cho E, Rimm EB, Stampfer MJ, et al. The impact of diabetes mellitus and prior myocardial infarction on mortality from all causes and from coronary heart disease in men. J Am Coll Cardiol 2002;40:954-60.

6 Saely $\mathrm{CH}$, Aczel S, Marte T, et al. Cardiovascular complications in type 2 diabetes mellitus depend on the coronary angiographic state rather than on the diabetic state. Diabetologia 2004;47:145-6.

7 Saely $\mathrm{CH}$, Schindewolf M, Zanolin D, et al. Single and combined effects of peripheral artery disease and of type 2 diabetes mellitus on the risk of cardiovascular events: a prospective cohort study. Atherosclerosis 2018;279:32-7.

8 Narkiewicz K, Redon J. 2013 ESH/ESC guidelines for the management of arterial hypertension. Eur Heart J 2013;34:2159-219.

9 Professional Practice Committee: Standards of Medical Care in Diabetes-2018. Diabetes Care 2018;41:S3.

10 Saely $\mathrm{CH}$, Aczel S, Marte T, et al. The metabolic syndrome, insulin resistance, and cardiovascular risk in diabetic and nondiabetic patients. J Clin Endocrinol Metab 2005;90:5698-703.

11 Drexel H, Aczel S, Marte T, et al. Is atherosclerosis in diabetes and impaired fasting glucose driven by elevated LDL cholesterol or by decreased HDL cholesterol? Diabetes Care 2005;28:101-7.

12 Levey AS, Stevens LA, Schmid CH, et al. A new equation to estimate glomerular filtration rate. Ann Intern Med 2009;150:604.

13 Berger A, Simpson A, Bhagnani T, et al. Incidence and cost of major adverse cardiovascular events and major adverse limb events in 
patients with chronic coronary artery disease or peripheral artery disease. Am J Cardiol 2019;123:1893-9.

14 Subherwal S, Patel MR, Kober L, et al. Peripheral artery disease is a coronary heart disease risk equivalent among both men and women: results from a nationwide study. Eur J Prev Cardiol 2015;22:317-25.

15 Criqui $\mathrm{MH}$, Aboyans V. Epidemiology of peripheral artery disease. Circ Res 2015;116:1509-26.

16 Pastori D, Pignatelli P, Sciacqua A, et al. Relationship of peripheral and coronary artery disease to cardiovascular events in patients with atrial fibrillation. Int J Cardiol 2018;255:69-73.

17 Hussein AA, Uno K, Wolski K, et al. Peripheral arterial disease and progression of coronary atherosclerosis. J Am Coll Cardiol 2011;57:1220-5.

18 Olesen KKW, Madsen M, Lip GYH, et al. Coronary artery disease and risk of adverse cardiac events and stroke. Eur J Clin Invest 2017;47:819-28.

19 Gyldenkerne C, Olesen KKW, Madsen M, et al. Extent of coronary artery disease is associated with myocardial infarction and mortality in patients with diabetes mellitus. Clin Epidemiol 2019;11:419-28.

20 Özcan C, Deleskog A, Schjerning Olsen A-M, et al. Coronary artery disease severity and long-term cardiovascular risk in patients with myocardial infarction: a Danish nationwide register-based cohort study. Eur Heart J Cardiovasc Pharmacother 2018;4:25-35.
21 Drexel H, Amann FW, Beran J, et al. Plasma triglycerides and three lipoprotein cholesterol fractions are independent predictors of the extent of coronary atherosclerosis. Circulation 1994;90:2230-5.

22 Saely CH, Rein P, Vonbank A, et al. Type 2 diabetes and the progression of visualized atherosclerosis to clinical cardiovascular events. Int J Cardiol 2013;167:776-80.

23 Brandtner EM, Muendlein A, Leiherer A, et al. Serum parathyroid hormone predicts mortality in coronary angiography patients with type 2 diabetes. J Clin Endocrinol Metab 2020;105:dgaa512.

24 Shah B, Rockman CB, Guo Y, et al. Diabetes and vascular disease in different arterial territories. Diabetes Care 2014;37:1636-42.

25 Beckman JA, Paneni F, Cosentino F, et al. Diabetes and vascular disease: pathophysiology, clinical consequences, and medical therapy: Part II. Eur Heart J 2013;34:2444-52.

26 Kamil S, Sehested TSG, Carlson N, et al. Diabetes and risk of peripheral artery disease in patients undergoing first-time coronary angiography between 2000 and 2012 - a nationwide study. BMC Cardiovasc Disord 2019;19:234

27 Weerarathna TP, Herath M, Liyanage G, et al. Prevalence and associations of subclinical peripheral artery disease among patients with type 2 diabetes without clinical macrovascular disease. Int $J$ Prev Med 2019;10:106.

28 Mach F, Baigent C, Catapano AL, et al. 2019 ESC/EAS guidelines for the management of dyslipidaemias: lipid modification to reduce cardiovascular risk. Eur Heart J 2020;41:111-88. 